\title{
Ampliación de un ciclo de mejora para conseguir una mayor implicación del alumnado
}

SILVIA MARTELO LANDROGUEZ

Universidad de Sevilla, Departamento de Administración de Empresas y Marketing smartelo@us.es

ORCID: https://orcid.org/0000-0002-4447-1088

D.O.I.: http://dx.doi.org/10.12795/JDU.2018.i01.78

Pp.: 1394-1410

\section{Resumen}

Este trabajo trata de resumir la experiencia final de innovación docente dentro del marco de la Red de Formación e Innovación Docente (REFID) durante el primer cuatrimestre del curso 2018/2019. Para ello, se presenta el ciclo de mejora aplicado en un bloque de la asignatura Introducción a la Economía de la Empresa (Organización) del Grado de Administración y Dirección de Empresas. Como resultado de esta actuación, hemos encontrado mejoras en el aprendizaje y la implicación en el aula por parte del alumnado. Por tanto, la experiencia se valora como algo positivo. Además, se han obtenido opiniones favorables de los alumnos sobre los cambios aplicados en las sesiones implicadas en el ciclo de mejora.

Palabras clave: Introducción a la Economía de la Empresa (Organización); Grado en Administración y Dirección de Empresas; Docencia universitaria; Experimentación docente universitaria; Kahoot 


\section{Descripción del contexto}

El ciclo de mejora ha sido dirigido al alumnado de la asignatura Introducción a la Economía de la Empresa (Organización). Se trata de una asignatura obligatoria correspondiente al primer cuatrimestre del primer curso del grado de Administración y Dirección de Empresas en la Facultad de Ciencias Económicas y Empresariales. Dicha asignatura posee un total de 6 créditos y tiene tanto teoría como práctica. La intervención se ha llevado a cabo en un grupo de clase impartido en sesión de tarde. Dicho grupo tiene 80 alumnos matriculados, siendo una media de 40 alumnos los que asisten habitualmente a clase.

La intervención ha tenido lugar en el primer bloque práctico del programa de la asignatura. Hasta el momento, se habían tratado temas introductorios totalmente teóricos. La carga práctica de los temas en cuestión aumenta su dificultad de cara a los estudiantes. Se considera, por tanto, de suma importancia conseguir la implicación del alumnado y conseguir captar su atención para eliminar sus bloqueos y miedos ante la aparición de la práctica en la asignatura.

En concreto, la intervención se ha llevado a cabo durante 5 sesiones de dos horas cada una. El último ciclo de mejora realizado se llevó a cabo en esta misma asignatura en el curso 2017/2018. Dicho ciclo abarcó 3 sesiones de dos horas. En dicha ocasión, el ciclo también tuvo lugar en la parte práctica de la asignatura, pero a partir del segundo tema de la parte práctica. En esta ocasión, se ha abarcado la parte práctica desde el principio para intentar cambiar la percepción del alumnado y evitar que dejen de asistir a clase porque piensen que no van a ser capaces de resolver los problemas.

El resultado del primer ciclo de mejora realizado en el curso pasado fue muy satisfactorio. Tal y como los propios alumnos reconocieron, la participación en clase y la 
realización de actividades les permitió asimilar mejor los conceptos. En consecuencia, la idea es partir de los cambios llevados a cabo el curso 2017/2018 y, sobre esa base, realizar algunas modificaciones. En concreto, una actividad que no funcionó bien (al tratarse de una actividad que tenía que realizar el alumnado fuera del aula) ha sido eliminada y se han añadido otras actividades nuevas que serán descritas a continuación.

El resultado del ciclo realizado en el curso pasado también ha provocado una modificación en el modelo metodológico, cambiando el orden y dándole más importancia en el ciclo actual a la fase práctica durante las sesiones.

\section{Diseño previo del Ciclo de Mejora Docente}

\section{Mapa o red de contenidos}

El mapa de contenidos se muestra en la Figura 1. El contenido planificado para el ciclo de mejora son los bloques: Función de producción, Planificación de la producción, Gestión de inventarios y Gestión de proyectos (resaltados en amarillo), aunque en el mapa se han incluido otros contenidos de la asignatura para que se entienda su sentido de conjunto.

Jornadas de Formación e Innovación Docente del Profesorado | № 1 (2018) Esta obra se distribuye con la licencia Creative Commons 


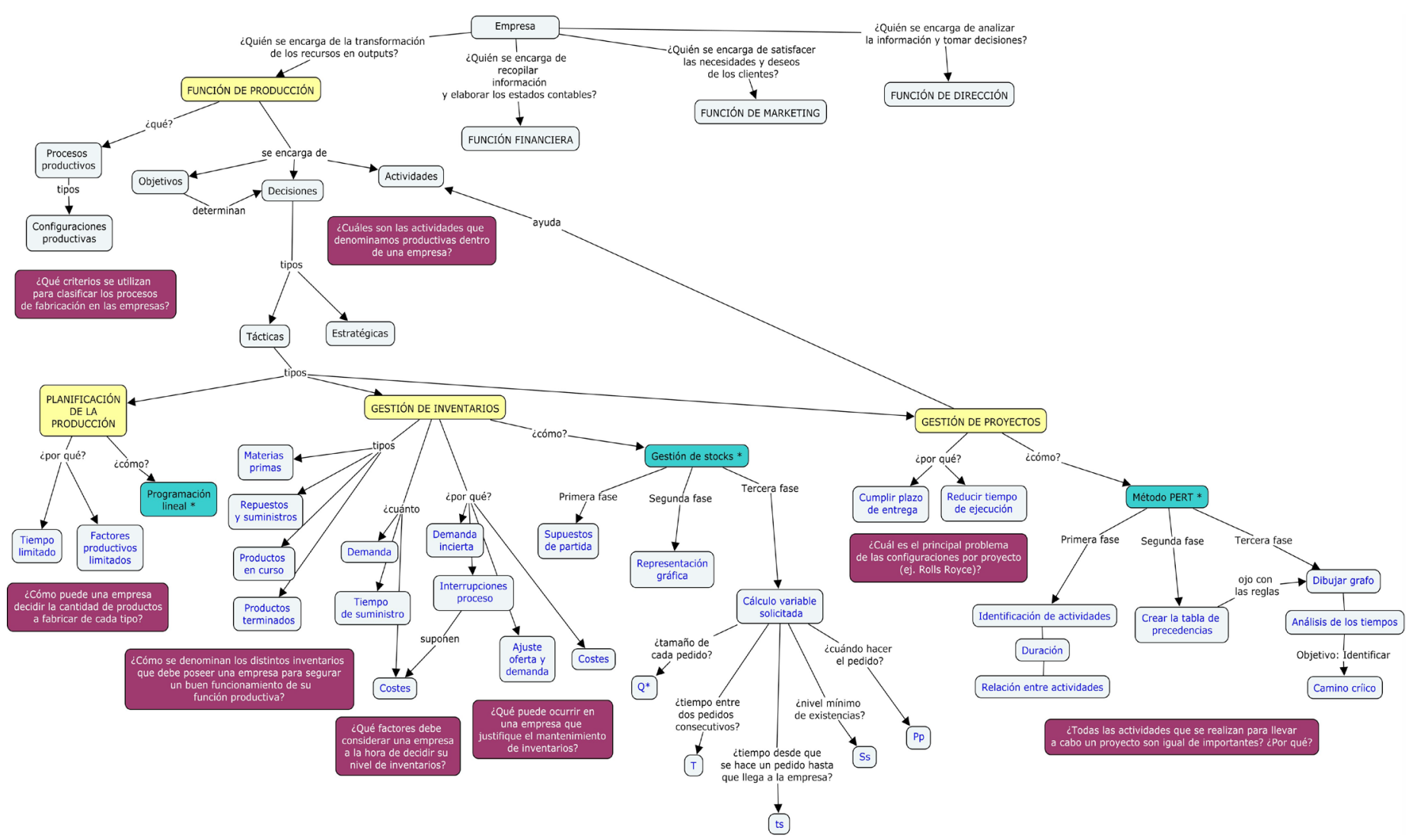

Figura 1. Mapa de contenidos

Jornadas de Formación e Innovación Docente del Profesorado | № 1 (2018)

(c) (i) $\odot$ Esta obra se distribuye con la licencia Creative Commons

Internacional (CC BY-NC-ND 4.0.) 
Dentro de cada uno de los bloques de contenido, hay una parte de contenidos procedimentales relacionados con la resolución de problemas (representados en verde). Las preguntas clave que son respondidas con el mapa de contenidos elaborado también aparecen recogidas en el mismo (en color morado).

Tras el análisis de las escaleras de aprendizaje en el ciclo anterior, se identificó como un posible problema que ciertos alumnos parecían no haber sido capaces de asociar algunas preguntas clave con los contenidos tratados en clase. Por esa razón, en los bloques Gestión de inventarios y Gestión de proyectos (que son comunes al ciclo anterior) se ha modificado la redacción de las preguntas. Asimismo, se va a intentar hacer mayor hincapié en las preguntas clave durante el desarrollo de las sesiones para que el alumnado relacione el cuestionario con los conceptos trabajados en clase.

\section{Modelo metodológico y secuencia de actividades}

El modelo metodológico tomado como base para planificar las actividades que se desarrollan en el ciclo de mejora es el siguiente:

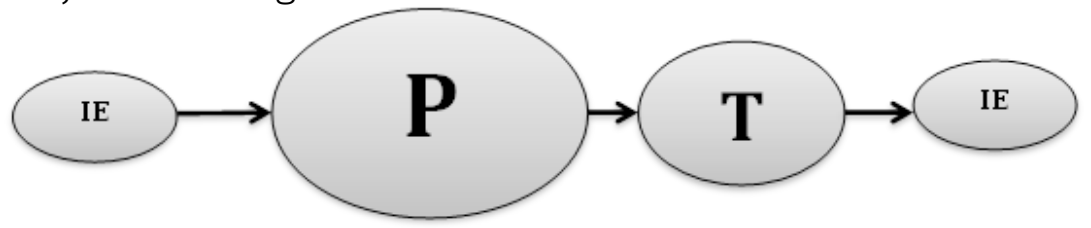

Figura 2. Modelo metodológico

IE (Ideas de los estudiantes): se dedicará una primera parte de cada sesión a una toma de contacto con los alumnos que permita saber qué saben ellos sobre el tema que vamos a tratar en clase ese día.

P (Práctica): a continuación, se realizará alguna actividad práctica antes de empezar a explicar los contenidos. El 
objetivo es que los alumnos vayan tomando conciencia de la utilidad de lo que van a estudiar y sepan para qué sirve. Se buscará un problema que se le pueda presentar a una empresa en el contexto real para captar así la atención de los estudiantes (Bain, 2007). Tanto la primera parte de la sesión como esta segunda pretenden fomentar la participación del alumno en el aula.

T (Teoria): en tercer lugar, el profesor explicará los contenidos principales del tema que se esté tratando que sean básicos para que los alumnos sigan el hilo de la asignatura. IE (Ideas de los estudiantes): Y serán los alumnos los que cerrarán la sesión a través de una evaluación de lo que han aprendido.

La secuencia de actividades propuesta se muestra en la siguiente tabla (Tabla 1):

\section{Tabla 1}

Secuencia de actividades del ciclo de mejora

\begin{tabular}{|c|c|c|c|}
\hline Fase del MM & Actividad & Contenidos básicos & Tiempo \\
\hline $\begin{array}{l}\text { Sesión 1: } \\
\text { Fase IE } \\
\text { inicial }\end{array}$ & $\begin{array}{l}\text { Cuestionario inicial ciclo } \\
\text { de mejora }\end{array}$ & & $15 \mathrm{~min}$ \\
\hline $\begin{array}{l}\text { Sesión 1: } \\
\text { Fase P }\end{array}$ & $\begin{array}{l}\text { Actividad con post-it. Esta } \\
\text { actividad tiene como ob- } \\
\text { jetivo fomentar la partici- } \\
\text { pación del alumnado en } \\
\text { clase y relacionar el conte- } \\
\text { nido de la asignatura con } \\
\text { el contexto real. }\end{array}$ & $\begin{array}{l}\text { Función de produc- } \\
\text { ción (Actividades) }\end{array}$ & $45 \mathrm{~min}$ \\
\hline $\begin{array}{l}\text { Sesión 1: } \\
\text { Fase T }\end{array}$ & Explicación teórica & $\begin{array}{l}\text { Función de produc- } \\
\text { ción (Actividades y } \\
\text { Objetivos) }\end{array}$ & $30 \mathrm{~min}$ \\
\hline $\begin{array}{l}\text { Sesión 1: } \\
\text { Fase IE final }\end{array}$ & $\begin{array}{l}\text { Actividad con post-it. Para } \\
\text { cerrar la sesión y compro- } \\
\text { bar si se ha entendido el } \\
\text { contenido. }\end{array}$ & & $15 \mathrm{~min}$ \\
\hline
\end{tabular}

Jornadas de Formación e Innovación Docente del Profesorado | № 1 (2018) Esta obra se distribuye con la licencia Creative Commons Reconocimiento-NoComercial-SinObraDerivada Internacional (CC BY-NC-ND 4.0.) 


\begin{tabular}{|c|c|c|c|}
\hline $\begin{array}{l}\text { Sesión 2: } \\
\text { Fase IE } \\
\text { inicial }\end{array}$ & $\begin{array}{l}\text { Actividad con fotos. Se } \\
\text { trata de una actividad para } \\
\text { evaluar la intervención en } \\
\text { el aula. }\end{array}$ & & $10 \mathrm{~min}$ \\
\hline $\begin{array}{l}\text { Sesión 2: } \\
\text { Fase P }\end{array}$ & $\begin{array}{l}\text { Vídeos. Consiste en la vi- } \\
\text { sualización de varios ví- } \\
\text { deos que muestran las } \\
\text { cuatro configuraciones } \\
\text { productivas para que los } \\
\text { alumnos identifiquen sus } \\
\text { características y sean ca- } \\
\text { paces de diferenciar en- } \\
\text { tre ellas. } \\
\quad \text { Preguntas a resolver: } \\
\text { 1) ¿Intervienen personas? } \\
\text { ¿ué proporción frente a } \\
\text { máquinas?, 2) ¿Qué da ori- } \\
\text { gen al funcionamiento del } \\
\text { proceso productivo?, 3) } \\
\text { ¿Cuántos productos dis- } \\
\text { tintos se fabrican?, 4) ¿Se } \\
\text { fabrican lotes? ¿De qué ta- } \\
\text { maño?, 5) ¿El proceso es } \\
\text { flexible o no?, 6) ¿Cómo } \\
\text { es el producto que se } \\
\text { fabrica? }\end{array}$ & $\begin{array}{l}\text { Función de pro- } \\
\text { ducción (procesos } \\
\text { productivos) }\end{array}$ & $45 \mathrm{~min}$ \\
\hline $\begin{array}{l}\text { Sesión 2: } \\
\text { Fase T }\end{array}$ & Explicación teórica. & $\begin{array}{l}\text { Función de produc- } \\
\text { ción (procesos pro- } \\
\text { ductivos y tipos de } \\
\text { decisiones) }\end{array}$ & $30 \mathrm{~min}$ \\
\hline $\begin{array}{l}\text { Sesión 2: } \\
\text { Fase IE final }\end{array}$ & $\begin{array}{l}\text { Vídeos. El objetivo es ver } \\
\text { la correspondencia entre } \\
\text { las características identifi- } \\
\text { cadas por los alumnos en } \\
\text { los vídeos y las caracterís- } \\
\text { ticas explicadas en clase. }\end{array}$ & & $20 \mathrm{~min}$ \\
\hline $\begin{array}{l}\text { Sesión 3: } \\
\text { Fase IE } \\
\text { inicial }\end{array}$ & $\begin{array}{l}\text { Kahoot. Actividad para } \\
\text { evaluar si los estudiantes } \\
\text { han entendido y recuerdan } \\
\text { los contenidos explicados } \\
\text { en la sesión anterior. }\end{array}$ & & $15 \mathrm{~min}$ \\
\hline
\end{tabular}

Jornadas de Formación e Innovación Docente del Profesorado I № 1 (2018) Esta obra se distribuye con la licencia Creative Commons Reconocimiento-NoComercial-SinObraDerivada Internacional (CC BY-NC-ND 4.0.) 


\begin{tabular}{|c|c|c|c|}
\hline $\begin{array}{l}\text { Sesión 3: } \\
\text { Fase P }\end{array}$ & $\begin{array}{l}\text { Se proyecta una transpa- } \\
\text { rencia con un problema al } \\
\text { que tiene que enfrentarse } \\
\text { una empresa y para resol- } \\
\text { verlo, los estudiantes tie- } \\
\text { nen que usar la técnica de } \\
\text { programación lineal. } \\
\text { Entre toda la clase, se } \\
\text { identifican las distintas va- } \\
\text { riables necesarias en el } \\
\text { problema y el profesor ex- } \\
\text { plica el funcionamiento de } \\
\text { la técnica apoyándose en } \\
\text { el problema. }\end{array}$ & $\begin{array}{l}\text { Planificación de la } \\
\text { producción (Progra- } \\
\text { mación lineal) }\end{array}$ & $45 \mathrm{~min}$ \\
\hline $\begin{array}{l}\text { Sesión 3: } \\
\text { Fase T }\end{array}$ & Explicación teórica. & $\begin{array}{l}\text { Planificación de la } \\
\text { producción (Progra- } \\
\text { mación lineal) }\end{array}$ & $30 \mathrm{~min}$ \\
\hline $\begin{array}{l}\text { Sesión 3: } \\
\text { Fase IE final }\end{array}$ & $\begin{array}{l}\text { Entre toda la clase, se } \\
\text { identifican los componen- } \\
\text { tes de la técnica explicada } \\
\text { sobre el problema previo } \\
\text { y se va recordando el fun- } \\
\text { cionamiento de la técnica } \\
\text { apoyándonos en la resolu- } \\
\text { ción del problema. }\end{array}$ & & $15 \mathrm{~min}$ \\
\hline $\begin{array}{l}\text { Sesión 4: } \\
\text { Fase IE } \\
\text { inicial }\end{array}$ & Kahoot & & $15 \mathrm{~min}$ \\
\hline $\begin{array}{l}\text { Sesión 4: } \\
\text { Fase P }\end{array}$ & $\begin{array}{l}\text { Se proyecta una transpa- } \\
\text { rencia con un problema al } \\
\text { que tiene que enfrentarse } \\
\text { una empresa y para resol- } \\
\text { verlo, los estudiantes tie- } \\
\text { nen que usar la técnica de } \\
\text { gestión de stocks. } \\
\text { Entre toda la clase, se } \\
\text { identifican las distintas va- } \\
\text { riables necesarias en el } \\
\text { problema y el profesor ex- } \\
\text { plica el funcionamiento de } \\
\text { la técnica apoyándose en } \\
\text { el problema. }\end{array}$ & $\begin{array}{l}\text { Gestión de inven- } \\
\text { tarios (Gestión de } \\
\text { stocks) }\end{array}$ & $50 \mathrm{~min}$ \\
\hline $\begin{array}{l}\text { Sesión 4: } \\
\text { Fase T }\end{array}$ & Explicación teórica & $\begin{array}{l}\text { Gestión de } \\
\text { inventarios }\end{array}$ & $30 \mathrm{~min}$ \\
\hline
\end{tabular}

Jornadas de Formación e Innovación Docente del Profesorado I № 1 (2018) Esta obra se distribuye con la licencia Creative Commons Reconocimiento-NoComercial-SinObraDerivada Internacional (CC BY-NC-ND 4.0.) 


\begin{tabular}{|c|c|c|c|}
\hline $\begin{array}{l}\text { Sesión 4: } \\
\text { Fase IE final }\end{array}$ & $\begin{array}{l}\text { Entre toda la clase, se re- } \\
\text { cuerdan los componentes } \\
\text { de la técnica explicada so- } \\
\text { bre el problema previo y el } \\
\text { funcionamiento de la téc- } \\
\text { nica apoyándonos en la } \\
\text { resolución del problema. }\end{array}$ & & $15 \mathrm{~min}$ \\
\hline $\begin{array}{l}\text { Sesión 5: } \\
\text { Fase IE } \\
\text { inicial }\end{array}$ & Kahoot & & $15 \mathrm{~min}$ \\
\hline $\begin{array}{l}\text { Sesión 5: } \\
\text { Fase P }\end{array}$ & $\begin{array}{l}\text { Se proyecta una transpa- } \\
\text { rencia con un problema } \\
\text { al que tiene que enfren- } \\
\text { tarse una empresa y para } \\
\text { resolverlo, los estudian- } \\
\text { tes tienen que usar la téc- } \\
\text { nica PERT. } \\
\text { Entre toda la clase, se } \\
\text { identifican las distintas va- } \\
\text { riables necesarias en el } \\
\text { problema y el profesor ex- } \\
\text { plica el funcionamiento de } \\
\text { la técnica apoyándose en } \\
\text { el problema. }\end{array}$ & $\begin{array}{l}\text { Gestión de proyec- } \\
\text { tos (Método PERT) }\end{array}$ & $45 \mathrm{~min}$ \\
\hline $\begin{array}{l}\text { Sesión 5: } \\
\text { Fase T }\end{array}$ & Explicación teórica & $\begin{array}{l}\text { Gestión de } \\
\text { proyectos }\end{array}$ & $30 \mathrm{~min}$ \\
\hline $\begin{array}{l}\text { Sesión 5: } \\
\text { Fase IE final }\end{array}$ & $\begin{array}{l}\text { Cuestionario final ciclo de } \\
\text { mejora y, de nuevo, activi- } \\
\text { dad de las fotos }\end{array}$ & & $15 \mathrm{~min}$ \\
\hline
\end{tabular}

\section{Seguimiento de la evolución de los modelos mentales de los estudiantes}

No tenemos que plantearnos sólo qué estamos enseñando a los estudiantes, también tenemos que plantearnos qué están aprendiendo (Finkel, 2008). Para evaluar el aprendizaje de los estudiantes, se ha realizado un cuestionario inicial y otro final con las mismas preguntas. El cuestionario inicial se realiza al principio de la primera sesión del ciclo y el cuestionario final en la última sesión del mismo. 
Las 6 preguntas que forman parte de dichos cuestionarios son las que aparecen en el mapa de contenidos en morado. Como recordatorio, las preguntas son las siguientes: 1) ¿Cuáles son las actividades que denominamos productivas dentro de una empresa?, 2) ¿Qué criterios se utilizan para clasificar los procesos de fabricación en las empresas?, 3) ¿Cómo puede una empresa decidir la cantidad de productos a fabricar de cada tipo?, 4) ¿Cómo se denominan los diferentes inventarios que debe poseer una empresa para asegurar un buen funcionamiento de su función productiva?, 5) ¿Qué factores debe considerar una empresa a la hora de decidir su nivel de inventarios?, 6) ¿Qué puede ocurrir en una empresa que justifique el mantenimiento de los inventarios?, 7) ¿Cuál es el principal problema de las configuraciones por proyecto (ej. Rolls Royce)?, 8) ¿Todas las actividades que se realizan para llevar a cabo un proyecto son igual de importantes? ¿Por qué?

\section{Aplicación del Ciclo de Mejora Docente}

\section{Relato resumido de las sesiones}

Sesión 1: Se entrega a cada uno de los estudiantes un cuestionario con las 8 preguntas incluidas en el mapa de contenidos (Fase IE inicial). Se les pide que se identifiquen de alguna forma (ya sea un símbolo, un número, etc.). Con esta actividad se pretende identificar dónde van a estar las dificultades de aprendizaje y así orientar mejor las siguientes sesiones. A continuación, se realiza una actividad con post-it que busca analizar los conocimientos que tiene el alumnado sobre el tema que se va a tratar ese día en clase (Fase P).

Los alumnos se dividen por grupos y tienen que pensar actividades que ellos creen que se llevan a cabo dentro 
de la función de producción de una empresa. Luego se les entrega unos post-it a cada grupo y tienen que apuntar una actividad en cada post-it. Cuando van terminando, una persona de cada grupo sale a la pizarra y pega los post-it. Luego salen varios alumnos a la pizarra y tienen que ordenar todos los post-it separándolos en grupos de actividades similares o relacionadas entre sí. A continuación, el profesor explica la función de producción, sus objetivos y las actividades/decisiones que forman parte de este subsistema (Fase T). Por último, se retoma la actividad con post-it y algunos alumnos salen a la pizarra, tienen que revisar los post-it pegados y quitar aquellos que recojan actividades que piensen que no corresponden a este subsistema de la empresa. Se hace una puesta en común entre el alumnado y el profesor (Fase IE final).

Sesión 2: Se empieza la sesión con una actividad de fotos (Fase IE inicial) que consiste en mostrar al alumnado 10 fotos que no tienen nada que ver con la asignatura (por ejemplo, la estela de un avión, un cactus, un túnel con luz al fondo, entre otras). El alumno tiene que elegir la foto que mejor represente sus emociones o sentimientos hacia el bloque del temario que vamos a empezar (es decir, la parte práctica). Al final del ciclo, se repetirá la actividad. El objetivo es identificar cambios en las emociones de los alumnos respecto a la parte considerada por ellos como más difícil en la asignatura antes y después de la intervención. A continuación, se visualizan cuatro vídeos. Cada uno de ellos muestra un proceso productivo distinto de los que se explicarán en clase. El objetivo es que asocien cada tipo de configuración con un ejemplo real para facilitar el recuerdo de las características de cada una de ellas. Mientras se visualiza cada vídeo, los alumnos tienen que tomar notas para contestar a varias preguntas que les van a permitir caracterizar cada uno de los procesos productivos y diferenciarlos entre sí (Fase P). Al tener que contestar las preguntas aumenta su atención. Una vez que han 
contestado a las preguntas, el profesor explica los contenidos del tema relacionados con los tipos de procesos productivos y los criterios utilizados para clasificarlos (Fase T). Para cerrar la sesión, se retoman las notas que tomaron los alumnos cuando vieron los vídeos y se va creando, entre todos, una tabla resumen de las características de las distintas configuraciones productivas y se compara con la tabla resumen que está incluida en las transparencias (Fase IE final). Sirve de repaso y al mismo tiempo sirve para detectar posibles confusiones y poder resolverlas sobre la marcha.

Sesión 3: Para evaluar si se ha entendido lo que se explicó en la clase anterior, se hace un Kahoot (Fase IE inicial). Los alumnos se implican bastante y al profesor le sirve para conocer el avance respecto al contenido que se va explicando en clase. A continuación, se proyecta una transparencia con un problema al que tiene que enfrentarse una empresa y para resolverlo, se tiene que usar la técnica de programación lineal. Los alumnos por grupos intentan resolverlo y luego hacemos una puesta en común (Fase P). Los alumnos tienen que leer el problema y pensar qué le pasa a la empresa. ¿Qué datos tiene la empresa? ¿Qué datos necesita? A continuación, se procede a explicar el objetivo de la técnica de programación lineal y sus componentes, y se les proporciona un guion con unos pasos a seguir para aplicar la técnica (Fase T). Antes de terminar la clase, se vuelve a la resolución del problema previa y se identifican los componentes de la técnica explicada recordando su funcionamiento (Fase IE final).

Sesión 4: Se empieza la clase haciendo un Kahoot (Fase IE inicial). Luego se muestra a los estudiantes una transparencia con un problema que se le puede presentar a una empresa en relación a su gestión de stocks (Fase P). Al igual que cuando se trató la otra técnica, entre toda la clase se van identificando las distintas variables necesarias en el problema y el profesor explica el funcionamiento de esta nueva técnica apoyándose en el problema. De esta 
forma, aumenta la implicación del alumnado en el aula. A continuación, se explican los contenidos básicos para entender el porqué de la gestión de inventarios y se profundiza en la técnica que vamos a utilizar y las variables necesarias (Fase T). Ahora se enlaza con la actividad inicial para relacionar las variables explicadas en la teoría con los datos identificados en el problema (Fase IE final). Los estudiantes siguen mejor la explicación. Al menos, son muchos los que participan.

Sesión 5: Se empieza la sesión con un Kahoot de repaso (Fase IE inicial). Antes de explicar la teoría de la siguiente técnica de resolución de problemas, se proyecta una transparencia con un problema que se le puede presentar a una empresa en relación a su gestión de proyectos. Hasta ahora siempre se había explicado toda la teoría primero, y luego el profesor hacía el problema como ejemplo al final. Al igual que en las sesiones anteriores, se ha cambiado el orden. Pienso que de esta forma el alumno está más implicado y más motivado porque deja de ser un sujeto pasivo en el aula. Los alumnos tienen que leer el problema y pensar qué le pasa a la empresa. ¿Qué datos tiene la empresa? ¿Qué datos necesita? (Fase P). Se percibe una alta participación de los estudiantes. Son capaces de identificar los datos que proporciona el enunciado del problema, aunque todavía no saben dar nombre a esos datos ni para qué van a servir. A continuación, el profesor explica los contenidos básicos para entender el porqué de la gestión de proyectos. Al igual que en la sesión anterior, el profesor se centra únicamente en explicar aquello que, por experiencia y según los cuestionarios iniciales, se detecta que es más complicado de entender. Con el apoyo de transparencias, se explica el objetivo de la técnica PERT y sus componentes. Asimismo, se les proporciona un guion con unos pasos a seguir para aplicar la técnica (fase T). Ahora se enlaza con el problema inicial y entre todos vamos relacionando las variables explicadas en la teoría con los datos identificados en el problema. Como cierre de la sesión y del ciclo de mejora, se pasa el cuestionario final (Fase IE final). Estas preguntas van a servir para evaluar el 
aprendizaje de los estudiantes. Se vuelve a hacer la actividad de las fotos para evaluar el ciclo de mejora realizado y los comentarios son bastante favorables.

\section{Evaluación del aprendizaje de los estudiantes}

Una vez realizado el análisis de los cuestionarios iniciales y finales, se elaboran las escaleras de aprendizaje. Las escaleras de aprendizaje permiten comparar el conocimiento inicial del alumnado con su conocimiento final. De esta forma, se puede analizar la evolución del alumnado en relación al contenido tratado en el aula.

En las escaleras de aprendizaje, los resultados se representan desde el modelo más alejado al más próximo a la realidad. A continuación, en las Figuras 3, 4 y 5, se muestran varios ejemplos basados en el análisis de tres de las preguntas que aparecen en los cuestionarios del ciclo de mejora realizado:

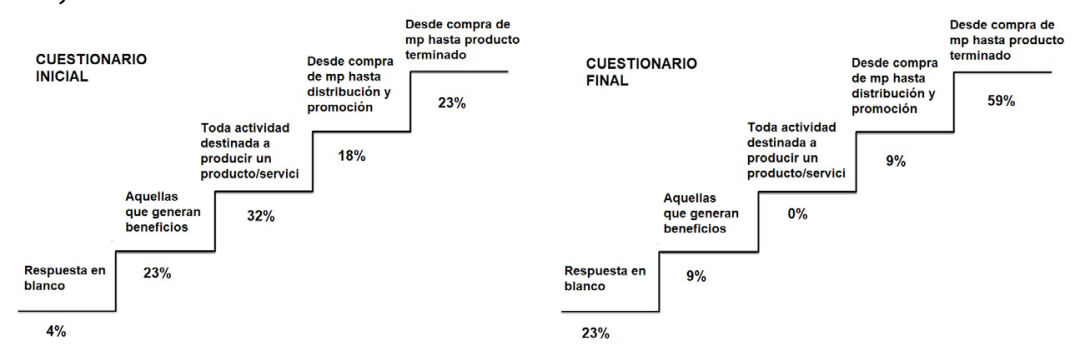

Figura 3. Escaleras de aprendizaje de la pregunta 1

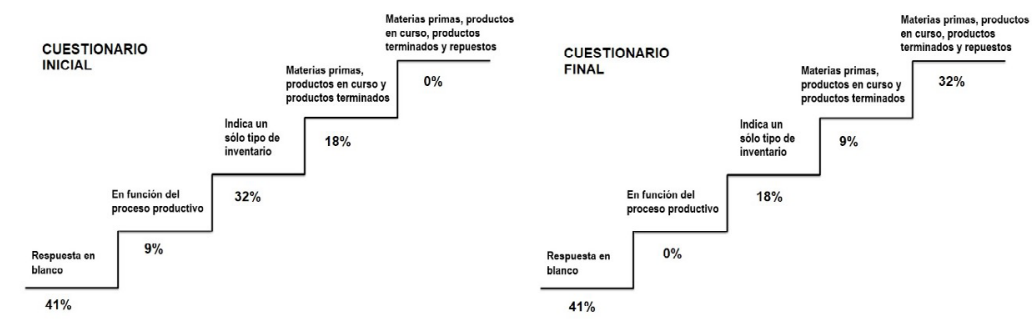

Figura 4. Escaleras de aprendizaje de la pregunta 4

Jornadas de Formación e Innovación Docente del Profesorado I № 1 (2018) Esta obra se distribuye con la licencia Creative Commons 


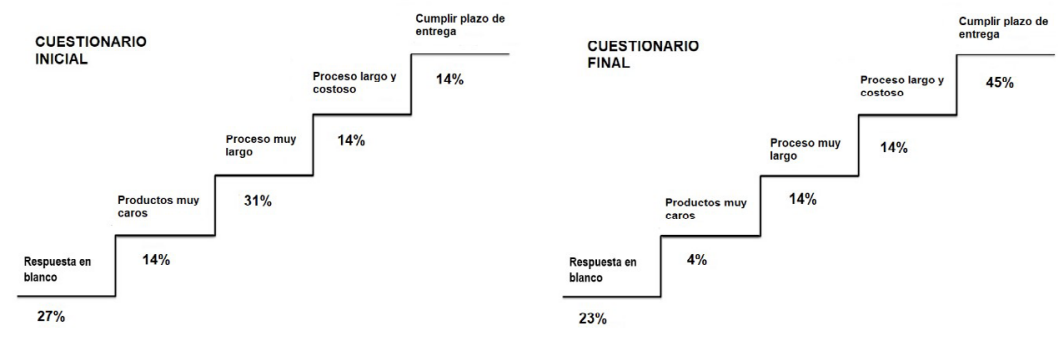

Figura 5. Escaleras de aprendizaje de la pregunta 7

Aunque en la mayoría de los casos se observa un avance hacia modelos más cercanos a la realidad, hay resultados más satisfactorios que otros. Destacaría el elevado número de respuestas en blanco. En esta ocasión, parece que se debe a que los estudiantes no se han tomado en serio la respuesta a los cuestionarios.

\section{Evaluación del Ciclo de Mejora Docente}

\section{Cuestiones a mantener y cambios a introducir para un futuro Ciclo de Mejora más amplio}

La utilización de las nuevas tecnologías en el aula ha funcionado muy bien, así que se seguirá manteniendo en futuros ciclos de mejora. En cambio, el alumnado sigue sin ser capaz de relacionar las preguntas clave incluidas en los cuestionarios inicial y final con los contenidos explicados en clase. Para futuros ciclos, se buscarán nuevas formas de enfocar las preguntas del cuestionario o hacer referencia a las mismas durante las distintas sesiones para ayudar a establecer relaciones.

Jornadas de Formación e Innovación Docente del Profesorado | № 1 (2018)

Esta obra se distribuye con la licencia Creative Commons 


\section{Aspectos de la experiencia que se pretenden incorporar a toda la práctica docente habitual}

La evaluación del ciclo se ha realizado principalmente a través del análisis de las impresiones percibidas en clase. El planteamiento de problemas parece resultar de ayuda a los estudiantes y despierta, en la mayoría de los casos, su interés y comprensión de la asignatura, sobre todo si se utilizan expresamente en el desarrollo de las sesiones. Tal y como los propios alumnos reconocen, la participación en clase y la realización de actividades les permite asimilar mejor los conceptos. Por tanto, se intentará incorporar estos cambios a la práctica docente habitual.

\section{Principios didácticos argumentados que han guiado la experiencia y que deben permanecer en el futuro}

La planificación y desarrollo del ciclo de mejora se ha basado en los siguientes principios didácticos:

- Colaboración y generación de conocimientos por parte de los estudiantes.

- El profesor como orientador y guía de los estudiantes, no como mero transmisor de información.

- No sólo es importante que los estudiantes entiendan los contenidos, es más importante si cabe que entiendan su utilidad y aplicación práctica.

- Elaboración de un mapa de contenidos que me permita identificar los contenidos estructurantes de la asignatura.

- El uso de las nuevas tecnologías en clase como forma de aumentar la motivación y participación de los estudiantes.

- Conocimiento de los modelos mentales de los estudiantes a través de cuestionarios. Análisis de sus respuestas e identificación de posibles obstáculos de aprendizaje. 


\section{Referencias bibliográficas}

Bain, K. (2007). Lo que hacen los mejores profesores universitarios. Valencia: Publicaciones de la Universidad de Valencia.

Finkel, D. (2008). Dar clase con la boca cerrada. Valencia: Publicaciones de la Universidad de Valencia.

Porlán, R. (2017). Enseñanza universitaria. Cómo mejorarla. Madrid: Ediciones Morata. 